\title{
Nanoimprint Lithography with UV-Curable Hyperbranched Polymer Nanocomposites
}

\author{
Valérie Geiser, Young-Hyun Jin, Yves Leterrier, * Jan-Anders E. Månson
}

Summary: Nano-scale patterns were produced with UV-curable acrylated hyperbranched polymer nanocomposites using nanoimprint lithography with a glass master in a rapid, low-pressure process. The pattern of the glass master was replicated with composites containing up to 25 vol\% $\mathrm{SiO}_{2}$ with a shape fidelity better than $98 \%$. Photo-rheology, interferometry and atomic force microscopy were used to analyze the material behavior. Attention was paid to the relationship between composition, nanoparticle dispersion, kinetics of photo-polymerisation, shrinkage, pressure and shape fidelity of nano-gratings. It was shown that the gel-point of the nanocomposite was an important factor that determined the stability as well as the dimensions of the imprinted structure. Dimensional accuracy also strongly depended on the level of internal stress, which in fact increased with the amount of silica. A resin rich layer on the surface of the composite accounted for the good surface quality of the nano-pattern.

Keywords: hyperbranched polymer; internal stress; nanocomposite; nanoimprint lithography; photo-polymerisation

\section{Introduction}

Nano-scale patterns are used in an increasing number of applications, ranging from optical sensors ${ }^{[1,2]}$ to transistors. ${ }^{[3]}$ Nanoimprint lithography (NIL) was first reported by $\mathrm{Chou}^{[4,5]}$ and is nowadays a well established contact lithography technique that allows for low-cost and high throughput production of nano-patterns and devices with sub$10 \mathrm{~nm}^{[6]}$ resolution. The efficiency of such devices is strongly dependent on the precision of the pattern, the reason for which dimensionally stable materials such as glass and silicon are often selected, in spite of high cost. Polymers are thus increasingly used as a low-cost alternative, ${ }^{[7,8]}$ however, their main drawback is the lack of dimensional stability ${ }^{[9,10]}$ due to excessive levels of internal stresses. Polymerisation shrinkage and cool-down from the process tempera-

Laboratoire de Technologie des Composites et Polymères (LTC), Ecole Polytechnique Fédérale de Lausanne (EPFL), 1015 Lausanne, Switzerland

E-mail: yves.leterrier@epfl.ch ture are major sources of residual stress. ${ }^{[11,12]}$ Combined with low stiffness, high coefficient of thermal expansion $(\mathrm{CTE})^{[13]}$ and surface tension driven viscous flow $^{[14]}$ these stresses lead to distortion and eventual decay of the nano-pattern.

Approaches towards stress reduction include photo-polymerisation ${ }^{[15,16]}$ with UV light, an extremely rapid crosslinking method that transforms liquid precursors into rigid solids within a few seconds. UV-polymerisation in combination with NIL was investigated in earlier studies. ${ }^{[17,18]}$ The process is carried out at room temperature and therefore thermal stresses which would result possible exothermic effects are usually negligible. Hyperbranched polymers (HBP) ${ }^{[19]}$ were also introduced in micro-engineering applications $^{[20]}$ due to their reduced polymerisation shrinkage and lower internal stress compared to standard resins, ${ }^{[21]}$ which proved to be a key feature for the production of a variety of microstructures with high dimensional accuracy. ${ }^{[2]}$ These materials are a low-cost alternative to dendrimers, ${ }^{[23]}$ with a less perfectly branched structure but same 
favorable behavior in terms of low Newtonian viscosity at high molecular weight. ${ }^{[24]}$ The introduction of a non-shrinking phase into the polymer matrix is a further route to reduce overall shrinkage. ${ }^{[25]}$ Geiser et al. ${ }^{[26]}$ have demonstrated a shrinkage reduction of $26 \%$ during photo-polymerisation upon addition of $20 \mathrm{vol} \%$ nano-sized $\mathrm{SiO}_{2}$ particles into an acrylated HBP matrix. ${ }^{[26]}$ The composite approach is attractive because it also improves the mechanical properties in terms of hardness, stiffness, scratch resistance, and coefficient of thermal expansion. ${ }^{[27-30]}$ However, the huge increase in viscosity due to the presence of nano-sized fillers often presents a challenge for processing. Moreover, solid particles with size comparable to nanostructures are likely to compromise the dimensional accuracy of these structures.

This work introduces UV-curable HBP nanocomposites for rapid and cost-effective fabrication of stable nano-structured devices with very high precision. The relationship between composition, polymerisation kinetics, shrinkage and shape fidelity of nano-gratings produced using low-pressure UV-molding were investigated. Attention was paid to the influence of the type of inorganic filler and pressure on the dimensional accuracy of the grating structures.

\section{Materials and Methods}

\section{Materials}

The monomer was based on a $3^{\text {rd }}$ generation hyperbranched polyether polyol, giving a 29functional hyperbranched polyether acrylate (Perstorp AB, Sweden). The photo-initiator was 1-hydroxy-cyclohexyl-phenyl-ketone (Irgacure ${ }^{\circledR}$ 184, Ciba Specialty Chemicals) at a concentration equal to $1 \mathrm{wt} \%$. It showed good solubility in the acrylate monomer. Two nanofillers were studied, both made of amorphous silica. Highlink ${ }^{\mathbb{B}}$ NanO G502 (Clariant) is a suspension of $30 \mathrm{wt} \%$ monodispersed $\mathrm{SiO}_{2}$ in isopropanol. The average particle size according to the supplier is $13 \mathrm{~nm}$, which corresponds to a specific surface area of about $230 \mathrm{~m}^{2} / \mathrm{g}$. X-ray disc centrifuge (BI-XDC, Brookhaven) measurements gave an average particle size of $23 \mathrm{~nm}$ with a standard deviation of $16 \mathrm{~nm}$. Aerosil ${ }^{\mathbb{R}} \quad$ R7200 (Degussa) is a $\mathrm{SiO}_{2}$ powder with a specific surface area of about $150 \mathrm{~m}^{2} / \mathrm{g}$ and a primary particle size of $12 \mathrm{~nm}$. Aerosil particles were subjected to surface treatment with methacrylsilane in order to promote interphase properties.

Composites containing up to $25 \mathrm{vol} \%$ $\mathrm{SiO}_{2}$ in the acrylated HBP were prepared and their properties are reported in Table 1. Details of sample preparation are given in an earlier article. ${ }^{[26]}$ The composites containing Highlink were true nanocomposites, where the inorganic phase was monodispersed in the polymer matrix. In contrast, the Aerosil powder could not be completely desagglomerated after ultrasound treatment, resulting in aggregates with an average size of $140 \mathrm{~nm}$. The dispersion state had a strong influence on the rheological behavior of the composites (Table 1).

\section{UV Lamp and Spectrometer}

A UV lamp with a $200 \mathrm{~W}$ mercury bulb (OmniCure 2000, Exfo, Canada) was used for all experiments. The light intensity was measured using a spectrometer (SolaCheck 2000, Solatell, UK) over a range of $270-470 \mathrm{~nm}$.

Table 1.

Properties of HBP nanocomposites with varying amounts of silica.

\begin{tabular}{lcccc}
\hline Property & Unit & & $\mathrm{SiO}_{2}$ & \\
& & 0 vol\% & 5 vol\% & 20 vol\% \\
\hline Viscosity (Aerosil/Highlink)[26] & $\mathrm{Pa}^{*} \mathrm{~s}$ & 4.6 & $7.3 / 60$ & $50 / 900$ '000 \\
Glass transition temperature[26] & ${ }^{\circ} \mathrm{C}$ & 9 & 9 & 9 \\
CTE (Highlink) & $10^{-6} /{ }^{\circ} \mathrm{C}$ & 118 & 114 & 84 \\
Water contact angle (Highlink) & & 45 & 78 & 72 \\
\hline
\end{tabular}




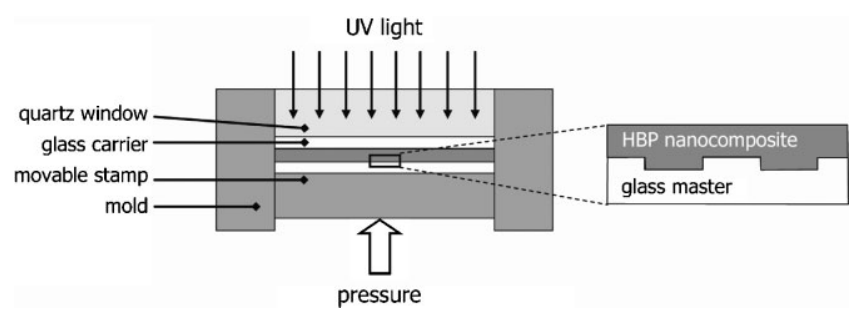

Figure 1.

Schematic drawing of the nanoimprint lithography tool.

\section{Nanoimprint Lithography (NIL)}

Figure 1 sketches the nanoimprint lithography tool that was designed and built for this study. It consists of a $8 \mathrm{~cm}$ diameter cylindrical steel mold equipped with a UVtransparent $3 \mathrm{~cm}$ thick quartz window. Pressure was applied using a pressurecontrolled pneumatic movable stamp to which was attached the glass master. Alignment accuracy was better than $0.02^{\circ}$.

The master was a dry etched glass grating (Figure 2) with a period of $360 \pm 1 \mathrm{~nm}$ and a depth of $12 \pm 1 \mathrm{~nm}$. This particular grating structure is used in wavelength interrogated optical sensors (WIOS) used for immuno-assay purposes as described in the work of Cottier et al. ${ }^{\text {[31] }}$

The material to imprint was dispersed on the master and covered with a glass slide the surface of which was activated by plasma treatment during $30 \mathrm{~s}$ with a high frequency generator (BD20V, Electro-technik products Inc.) for better adhesion of the polymer material. Pressure was applied while the material was polymerized through the quartz window. Approximately
$12 \%$ of UV-light was absorbed through the glass carrier. The UV-intensities reported in the following were measured under the glass carrier, i.e. on the HBP surface. After polymerisation the pressure was released and the master was removed from the imprinted material attached to the glass carrier. No special surface treatment was needed to help demolding, due to the $25^{\circ}$ clearance angle of the glass grating. The whole process sequence lasted less than $3 \mathrm{~min}$.

\section{Atomic Force Microscopy (AFM)}

The topography of the gratings, both attached and detached from the glass carrier, was analyzed by AFM (Multimode II, Veeco) in contact mode using a tip with a spring constant of $0.06 \mathrm{~N} / \mathrm{m} .512$ scans were recorded over a length of $2 \mu \mathrm{m}$ (Figure $2 \mathrm{~b}$ ) from which an average profile, such as shown in Figure 2, was calculated. In order to quantify the influence of the processing parameters on the fidelity of the imprinted gratings, the step height as well as the top and bottom dimensions as indicated a)

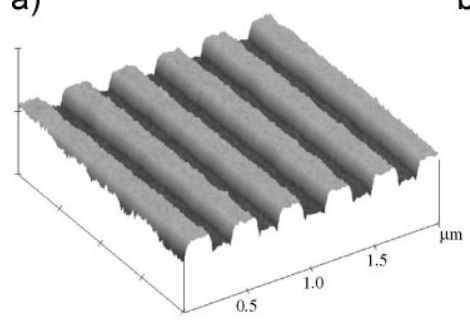

b)

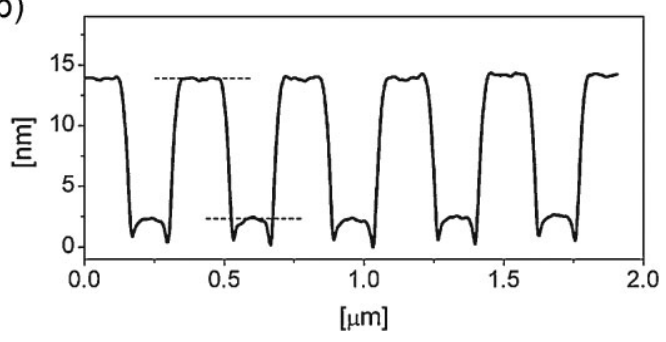

Figure 2.

(a) AFM surface plot and (b) averaged profile of the glass master grating. The troughs in the corners of the master grating resulted from the dry etching step during the production process. The step height was measured between the dashed lines. 
in Figure 3 were compared. Each dimension was averaged over 5 repeating elements.

\section{Photo-Rheology}

Real-time stiffness build-up during photopolymerisation of the HBP and HBP composites was measured on a straincontrolled rotational rheometer (ARES, Rheometric Scientific, 2kFT transducer) equipped with a UV set-up as described in Schmidt et al. ${ }^{[32]}$ Parallel plate geometry with diameter $8 \mathrm{~mm}$ was used at room temperature and $10 \mathrm{~Hz}$.

\section{Interferometry}

Real-time polymerisation shrinkage was measured with a Michelson interferometer on a $100 \mu \mathrm{m}$ thick sample film as described in a former article. ${ }^{[26]}$

\section{Thermal Mechanical and Stress Analysis}

The coefficient of thermal expansion was measured with a thermo-mechanical analyzer (TMA 402, Netsch) using a heating and cooling rate of $5^{\circ} \mathrm{C} / \mathrm{min}$. The thermal deformation of HBP and HBP composite films under a compression load of $1.3 \mathrm{~N}$ was recorded. It was veryfied that no creep phenomena occurred. The Young's modulus was measured with a dynamic mechanical analyzer (DMA Q800, TA Instruments) at $1 \mathrm{~Hz}$ and a maximum elongational strain of $0.15 \%$. The in-plane internal stress of HBP and HBP composite coatings was determined from the curvature of coated aluminum beams, and calculated according to the model of Inoue. ${ }^{[33]}$ The beam-bending set-up is described in detail in the work of Schmidt et al. ${ }^{[21]}$

\section{Contact Angle Measurements}

Water contact angles were measured using a contact angle meter (Digidrop, GBX) at room temperature, using deionized-grade water.

\section{Results}

Figure 4 shows the averaged profiles of the photo-cured gratings for different UVillumination times, UV-light intensities, pressures and filler fractions. It is evident that good replication fidelity was achieved whatever the process conditions. After only 3 seconds of illumination, or using a low intensity of $5 \mathrm{~mW} / \mathrm{cm}^{2}$, a stable grating structure was observed after lift-off of the master (Figure 4a,b). Even at the minimum pressure of 1 bar the grating was imprinted with good quality (Figure 4c). The grating structure was also properly imprinted in the composite material up to the highest filler content (Figure $4 d$ ). In all cases the grating period was preserved, even when the gratings were removed from the glass carrier, and the "ears" corresponding to the master troughs were present. Figure 5 shows the top and bottom dimensions as well as the step height for the same conditions, and confirms that the period of the reproduced gratings differed by less than $2 \%$ from the period of the master under all conditions, i.e. the shape fidelity

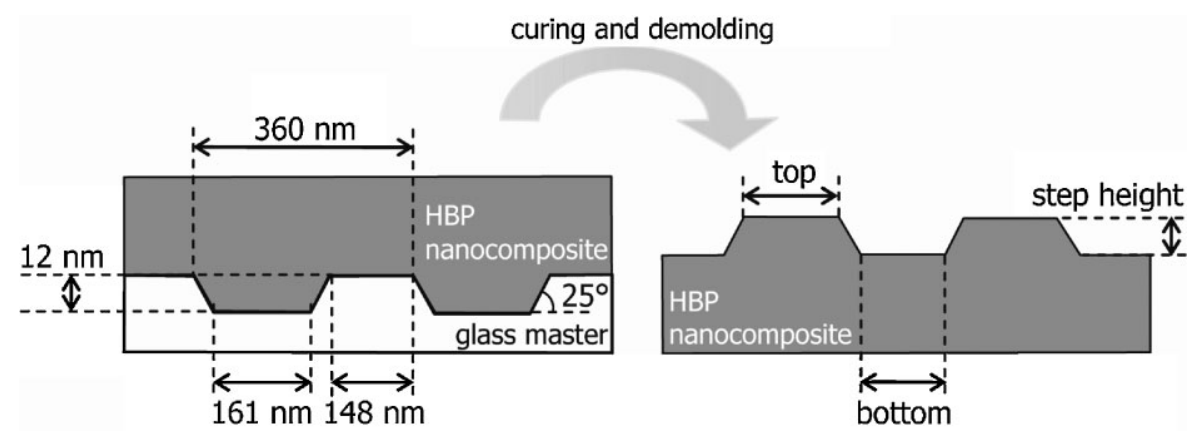

Figure 3.

Dimensions of the master grating and nomenclature of the dimensions of the imprinted grating. 


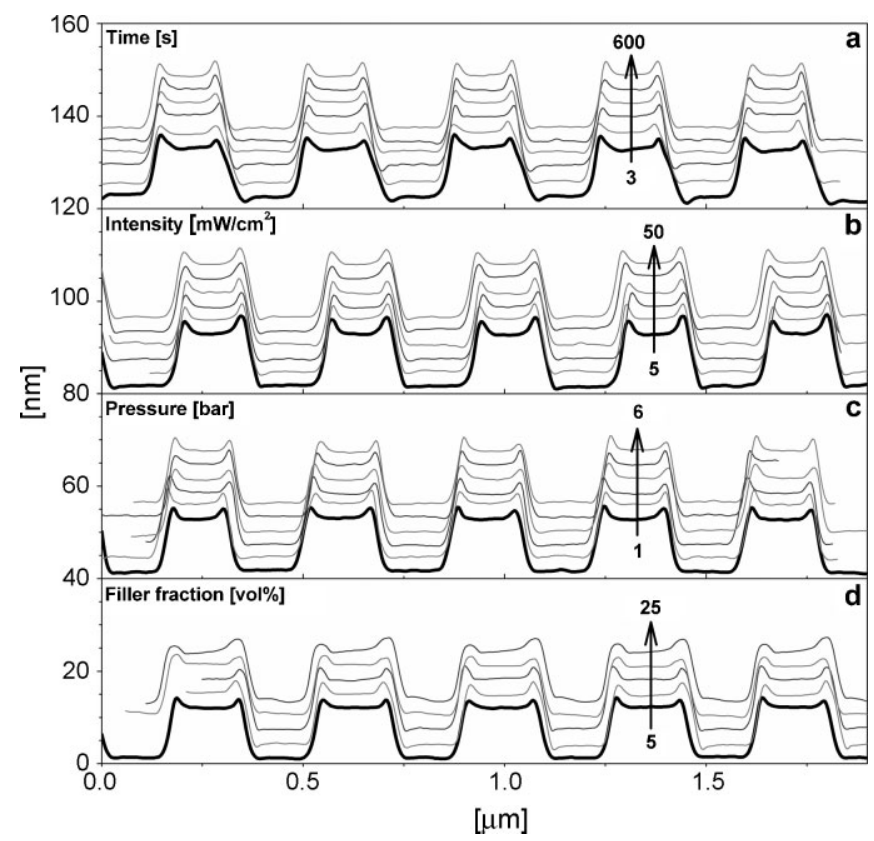

Figure 4.

Averaged AFM profiles of HBP and HBP nanocomposite gratings. Individual profiles are offset for legibility. (a) HBP gratings as a function of illumination time. Intensity $=25 \mathrm{~mW} / \mathrm{cm}^{2}$, pressure $=5$ bar. (b) HBP gratings as a function of UV-intensity. Time $=600 \mathrm{~s}$, pressure $=5$ bar. (c) $\mathrm{HBP}+5$ vol\% $\mathrm{SiO}_{2}$ (Highlink) gratings as a function of pressure. Intensity $=60 \mathrm{~mW} / \mathrm{cm}^{2}$, time $=90 \mathrm{~s}$. (d) HBP composite gratings as a function of filler (Highlink) fraction. Intensity $=60 \mathrm{~mW} / \mathrm{cm}^{2}$, time $=90 \mathrm{~s}$.

was better than $98 \%$. When looking at the measured grating dimensions, it is evident that during 10 seconds the step height remained constant within experimental scatter and then decreased gradually (Figure 5a). The intensity did not influence the dimensions of the imprinted grating (Figure 5b). However, while the top and bottom dimensions correlated with the corresponding master dimensions, the step height was reduced by $4.3 \pm 1.3 \%$ with respect to the master step height. Pressure levels ranging from 1 to 6 bar were applied to HBP nanocomposites containing 5 vol\% $\mathrm{SiO}_{2}$. For both types of composite (Highlink and Aerosil) the dimension of the bottom part was reduced, and that of the top part was expanded with respect to the corresponding dimensions on the master. This interesting result is examined in the following section. Moreover, the step height of the imprinted grating was $9.4 \%$ smaller than the master step height (Figure 5c).
The bottom and top dimensions, as well as the step height, strongly depended on the amount of filler (Figure 5d). Similarly to the effect of pressure, the top part was expanding, while the bottom part and the step height were reduced. The clearance angle of the grating did not systematically change and only varied within $6 \%$ of the clearance angle of the master grating. The "ears" on the grating edges became less sharp, but the surface finish of the gratings was not compromised by the presence of the silica. No difference was observed between Highlink and Aerosil composites.

\section{Discussion}

It is remarkable, that at a pressure as low as 1 bar the grating structure was imprinted in nano-composites containing up to $25 \mathrm{vol} \%$ silica, despite the high viscosity that these materials exhibit. The period of the HBP 

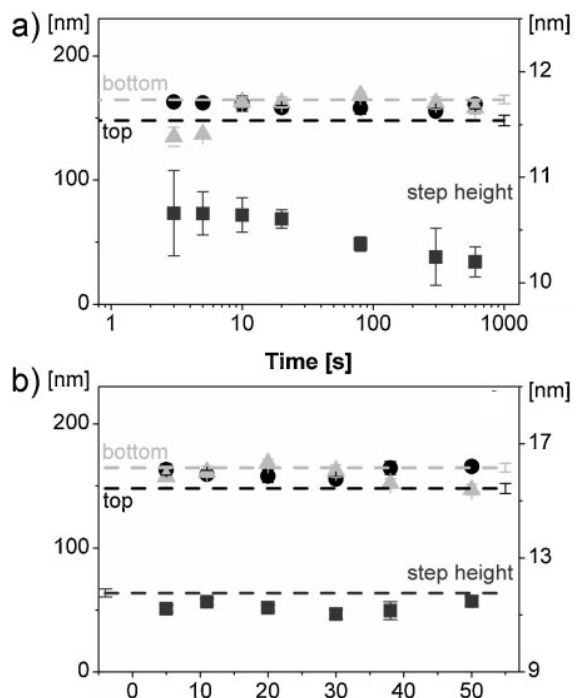

nanocomposite gratings being equal to that of the glass master, this is a promising result for the production of polymer WIOS by nano-imprint lithography, because for good performance of the optical sensing devices, the period of the grating is the key parameter to control. However, shrinkage of the step height occurred especially at high filler loading, and a unexpected lateral expansion of the grating walls was observed. These two phenomena resulted from the dynamics of structure build-up during cure of the polymer confined in the grating structure, including gelation and internal stress. Figure 6 synthesizes a NIL process including these two key events and is described in the following sections.
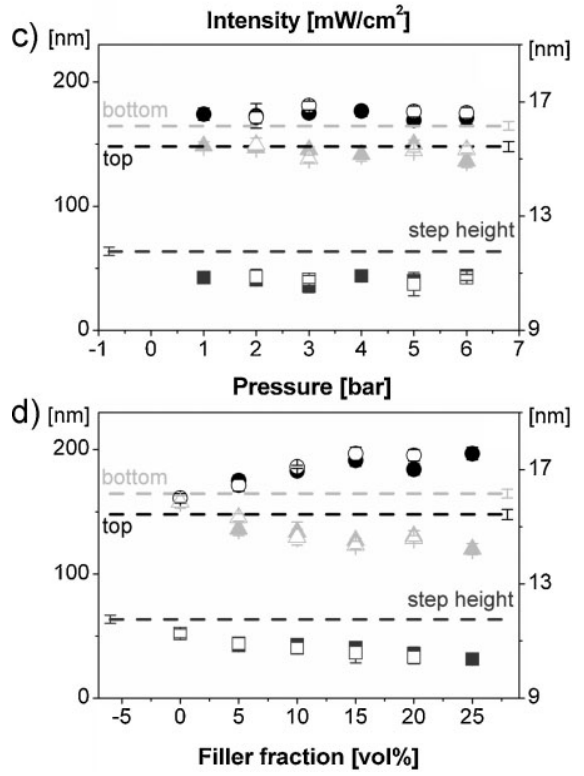

\section{Gelation}

Gelation being the transition between liquid and solid material, is an important parameter that determines the stability of the gratings. The gel-point was determined with photo-rheology from the crossover of storage and loss moduli, $\mathrm{G}^{\prime}$ and $\mathrm{G}^{\prime \prime}$, and was found to occur just below 3 seconds for $\mathrm{HBP}$ at an intensity of $25 \mathrm{~mW} / \mathrm{cm}^{2}$. At that point the double-bond conversion reached $15 \%{ }^{[26]}$ and the shear modulus was approximately $1 \mathrm{MPa}$ (Figure 7), hence the stability of the imprinted HBP grating after only 3 seconds of illumination (Figure $4 a$ ). The influence of gelation on step height shrinkage is analyzed in Figure 8. The linear shrinkage, calculated from the reduction of the step height with respect to the step height at 3 seconds, was delayed in time by a factor of about 10 , compared with the linear shrinkage determined by interferometry on flat HBP films. As polymerisation set in, the equilibrium volume of the HBP reduced (step 1 to step 2 in Figure 6). In the absence of external pressure, voids would have immediately developed in the pits of the master grating. However, under constant external pressure the viscous material continued to fill the grating cavities (step 2 in Figure 6). Only once the material had gelled, plastic flow was no longer possible and further polymerisation shrinkage led to the observed reduction of the step height 


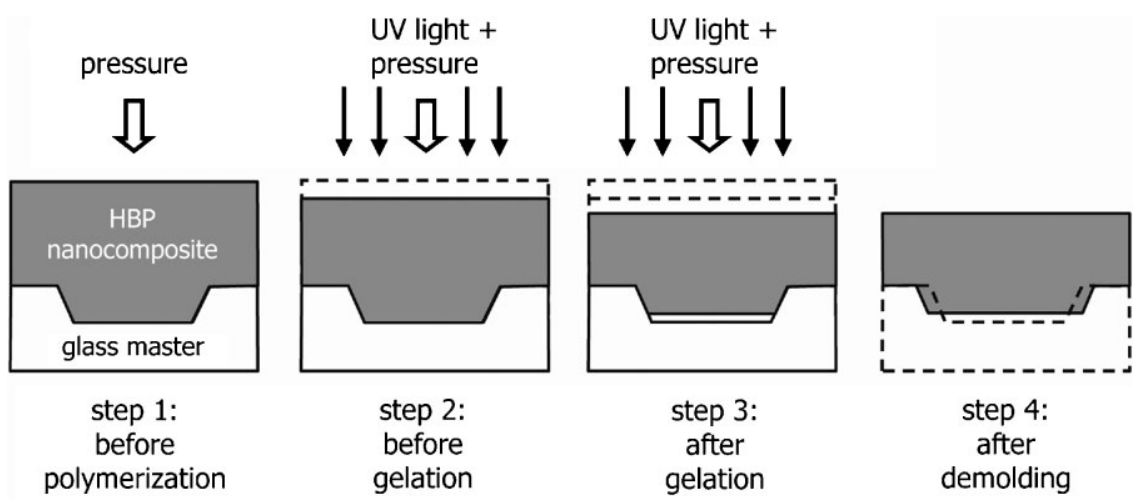

Figure 6.

Schematic drawing the grating formation process during NIL.

(step 3 in Figure 6). While the gel-point determined using photo-rheology was around 3 seconds, gelation determined by kinetic analysis occurred at 5 seconds $^{[26]}$ for the current system. The latter result corresponds well with the moment after which the step height started shrinking.

The reason why the intensity did not influence the dimensions of the imprinted gratings, is because the maximum conversion is independent of the UV-light intensity. ${ }^{[26]}$ Several studies confirmed that shrinkage is related to conversion for non-vitrifying systems. ${ }^{[34-36]}$ The glass transition temperature $\mathrm{T}_{\mathrm{g}}$ being equal to $9^{\circ} \mathrm{C}^{[26]}$ for the HBP and the composites independent of composition, the present systems indeed did not vitrify. The average shrinkage determined from the reduction in step height of the polymer grating with respect to the master step height $(4.3 \pm 1.3 \%)$ was

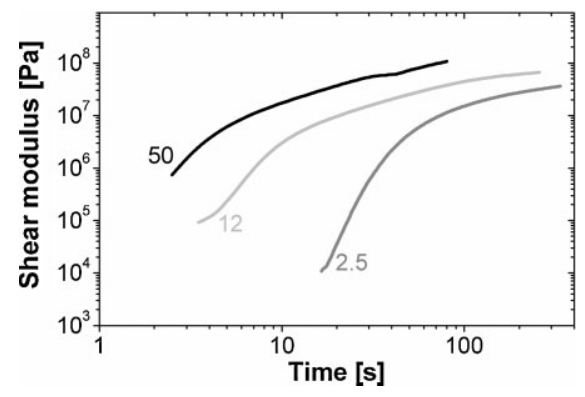

Figure 7.

Shear modulus of HBP as a function of time under different UV-light intensities ( $\mathrm{mW} / \mathrm{cm}^{2}$, as indicated). equal within experimental scatter to the linear shrinkage measured on flat films $(4.6 \pm 0.5 \%)$.

\section{Internal Stress}

A surprising result was the inversion of the bottom and top dimensions which was evident when the pressure, or the filler fraction, were increased (Figure 5c,d). The sidewalls of the master were tilted by an angle of approximately $25^{\circ}$, the lateral dimensions of the grating were therefore a function of the step height, which in fact depended on the amount of silica in the composite. As an example, the step height of the imprinted grating was reduced by $12 \%$ with respect to the master grating upon addition of $25 \mathrm{vol} \% \mathrm{SiO}_{2}$. This would

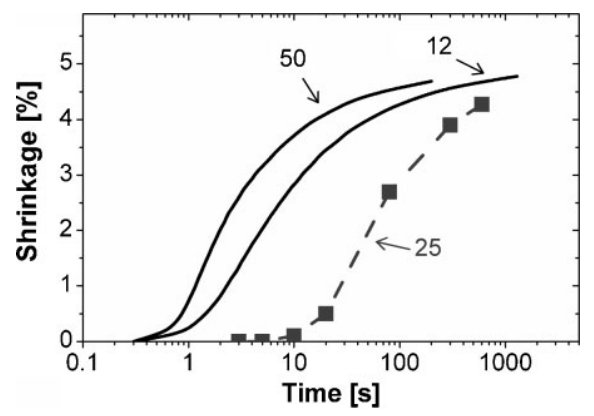

Figure 8.

Linear polymerisation shrinkage as a function of illumination time and UV-intensity $\left(\mathrm{mW} / \mathrm{cm}^{2}\right.$, as indicated) measured on HBP film (solid line) compared to the shrinkage as determined from the reduction in grating step height (squares and dotted line). 
have increased the top part by $6 \mathrm{~nm}$, however, the real increase was $49 \mathrm{~nm}$. The fact that the silica reduced the step height (Figure 5d) was surprising as well, since it had been shown that the presence of an inorganic filler reduced the overall polymerisation shrinkage. ${ }^{[26]}$ The deviation of the top and bottom dimensions of the imprinted grating from the master dimensions was due to internal stress effects. Polymerisation shrinkage and simultaneous stiffness build-up are the reason for internal stress build-up in polymer materials. Even though the presence of silica reduced the amount of polymerisation shrinkage, ${ }^{[26]}$ the internal stress increased linearly with the filler fraction, due to the increased Young's modulus of the composites (Figure 9). Highlink and Aerosil composites showed the same level of internal stress, despite the difference in Young's modulus resulting from difference in particle dispersion. In fact, the reduced stiffness of the Aerosil composites was counteracted by the increased polymerisation shrinkage. ${ }^{[26]}$ The internal stress measurements as shown in Figure 9 were done on flat films that were constrained in two dimensions by the substrate, i.e. under plane stress conditions. In the case of gratings the constrains were in three dimensions, i.e. under hydrostatic conditions, hence higher stress levels ${ }^{[37,38]}$ especially when the polymer was processed

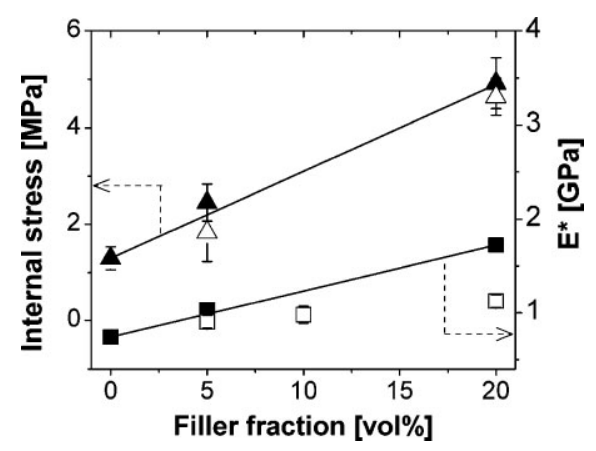

\section{Figure 9.}

Internal stress and Young's modulus of HBP nanocomposites as a function of filler fraction and polymerized with UV-intensity equal to $50 \mathrm{~mW} / \mathrm{cm}^{2}$. Closed symbols: Highlink composites. Open symbols: Aerosil composites. above its ultimate $\mathrm{T}_{\mathrm{g}}$. After lift-off of the master the stress relaxation led to the observed deformation of the grating (step 4 in Figure 6).

Nanoparticles increased the stress within the grating structures, however, they did not compromise the surface quality, the reason of which was found to be a resin rich layer a the surface of the device (Figure 10). Deformation of the composite material under pressure led to exudation of the HBP phase, as often observed in case of compression molding of reinforced polymers in narrow geometries. ${ }^{[39]}$

To summarize, the grating formation process during NIL started with loading the liquid precursor on the glass master (step 1 in Figure 6). Upon application of pressure the materials filled out the master grating cavities. At this stage exudation of the polymer took place, thus ensuring a good surface quality. As polymerisation was initiated, shrinkage occurred in the entire volume of the precursor, and the viscous material continued to fill the grating cavities under the constand applied pressure (step 2 in Figure 6). After gelation plastic flow was no longer possible and further polymerisation shrinkage led to reduction of the step height and internal stress started to build up (step 3 in Figure 6). After cure completion and release of the pressure the replicated grating was demolded and internal stresses could relax, resulting in the observed deformation of the grating structure (step 4 in Figure 6).

Considering that small changes in internal stress have a considerable influence on the geometry of a nano-pattern, the present low-stress HBP nanocomposites show immense advantage over commercial UVcurable acrylates, where the stress level goes up to $16 \mathrm{MPa} \cdot{ }^{[40,41]}$ In the standard photoresists SU-8 stresses are around $20 \mathrm{MPa}^{[42]}$ before and up to $75 \mathrm{MPa}^{[43]}$ after postexposure bake. A further advantage for the production of stable nano-patterns is the reduced CTE (Table 1) for better thermal stability. Moreover, the contact angle increased from $45^{\circ}$ for the pure HBP to $73^{\circ}$ for the composite containing $20 \mathrm{vol} \%$ 

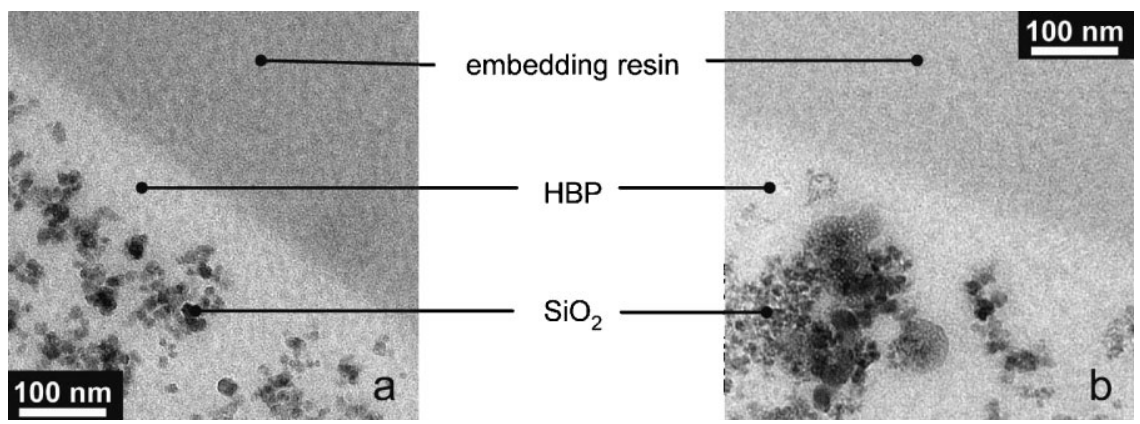

Figure 10.

Transmission electron micrographs of grating surfaces of HBP nano-composites containing 5 vol\% $\mathrm{SiO}_{2}$ from (a) Highlink and (b) Aerosil. A HBP resin rich layer is evident in both cases.

Highlink (Table 1), indicating that the composite was hydrophobic while the HBP was rather hydrophilic. The hydrophobicity of the Highlink composites was most likely due to the surface treatment of the silica particles, the nature of which was not known.

The HBP and HBP nanocomposites enabled nanoscale gratings with an exceptional fidelity to be replicated using a low pressure NIL method and very short process time. Since these patterns were obtained for a broad range of process conditions and of compositions, the present results should be useful to produce a variety of nano-sized patterns for a vast range of device applications.

\section{Conclusion}

Nano-sized gratings were produced from UV-curable acrylated HBP nano-composites with up to $25 \mathrm{vol} \%$ silica by nanoimprint lithography in rapid low-pressure process using a glass master. A pressure as low as 1 bar and short process time of 3 seconds were sufficient to imprint a stable grating into the high viscosity composite material with a shape fidelity better than $98 \%$. The period of the composite gratings was equal to the one of the master, but the step height depended on the time at gelation, and the lateral dimensions depended on the level of internal stress. Internal stress increased with the amount of silica, independent of the dispersion state. Pressure and UV-light intensity did not influence the grating dimensions provided that the maximum acrylate conversion was achieved. The surface quality of the grating was not compromised by the presence of the silica, due to the exudation of HBP resin rich surface layer.

Acknowledgements: The authors gratefully acknowledge financial support from the Swiss National Science Foundation (SNF project \# 200020-111706). They would like to thank Perstorp AB for the supply of polymer samples, Max Wiki from Dynetix Biosystems for the supply of the glass master and for useful advice and Yannick Roulin for the design of the NIL tool.

[1] M. Wiki, R. E. Kunz, Opt. Lett. 2000, 25, 463.

[2] F. C. Chien, C. Y. Lin, J. N. Yih, K. L. Lee, C. W. Chang, P. K. Wei, C. C. Sun, S. J. Chen, Biosens. Bioelectron. 2007, 22, 2737.

[3] X. Cheng, D. W. Li, L. J. Guo, Nanotechnology 2006, 17, 927.

[4] S. Y. Chou, P. R. Krauss, P. J. Renstrom, Appl. Phys. Lett. 1995, 67, 3114.

[5] S. Y. Chou, P. R. Krauss, P. J. Renstrom, 40th International Conference on Electron, Ion, and Photon Beam Technology and Nanofabrication (EIBPN), Atlanta, Ga, May 28-31 1996, pp. 4129.

[6] M. D. Austin, H. X. Ge, W. Wu, M. T. Li, Z. N. Yu, D. Wasserman, S. A. Lyon, S. Y. Chou, Appl. Phys. Lett. 2004, 84, 5299.

[7] M. Csete, A. Kohazi-Kis, V. Megyesi, K. Osvay, Z. Bor, M. Pietralla, O. Marti, Org. Electron. 2007, 8, 148. [8] A. Kocabas, A. Aydinli, Opt. Express 2006, 14, 10228. 
[9] E. Buck, K. Petersen, M. Hund, G. Krausch, D. Johannsmann, Macromolecules 2004, 37, 8647.

[10] Y. Ding, H. W. Ro, T. A. Germer, J. F. Douglas, B. C. Okerberg, A. Karim, C. L. Soles, ACS Nano 2007, online. [11] C. N. Bowman, N. A. Peppas, Macromolecules 1991, 24, 1914.

[12] J. Lange, S. Toll, J. A. E. Månson, A. Hult, Polymer 1995, 36, 3135.

[13] Y. Hirai, S. Yoshida, N. Takagi, 47th International Conference on Electron Ion and Photon Beam Technology and Nanofabrication (EIPBN), Tampa, Florida, May 27-30 2003, pp 2765.

[14] M. Hamdorf, D. Johannsmann, J. Chem. Phys. 2000, 112, 4262.

[15] L. E. Schmidt, Y. Leterrier, D. Schmah, J. A. E. Månson, D. James, E. Gustavsson, L. S. Svensson, J Appl. Polym. Sci. 2007, 104, 2366.

[16] E. Andrzejewska, Prog. Poly. Sci. 2001, 26, 605.

[17] J. H. Choi, S. U. Jung, D. G. Choi, J. H. Jeong, E. S. Lee, IEEE International Symposium on Advanced Gate Stack Technology (ISAGST), Austin, TX, 2006 2008, pp 195.

[18] J. Lee, K. Choi, G. Kim, S. Lee, Microelectron. Eng. 2007, 84, 963.

[19] C. J. Hawker, R. Lee, J. M. J. Frechet, J. Am. Chem. Soc. 1991, 113, 4583.

[20] Y. H. Jin, Y. H. Cho, L. E. Schmidt, Y. Leterrier, J. A. E. Månson, J. Micromech. Microeng. 2007, 17, 1147.

[21] L. E. Schmidt, D. Schmah, Y. Leterrier, J. A. E. Månson, Rheol. Acta. 2007, 46, 693.

[22] L. E. Schmidt, S. Yi, Y. H. Jin, Y. Leterrier, Y. H. Cho, J. A. E. Månson, J. Micromech. Microeng. 2008, 18, 45022.

[23] A. W. Bosman, H. M. Janssen, E. W. Meijer, Chem. Rev. 1999, 99, 1665.

[24] J. M. J. Frechet, C. J. Hawker, I. Gitsov, J. W. Leon, J. Macromol. Sci. Pure Appl. Chem. 1996, A33, 1399.
[25] M. Atai, D. C. Watts, Dent. Mater. 2006, 22, 785.

[26] V. Geiser, Y. Leterrier, J.-A. E. Månson, J Appl. Polym. Sci. 2009, 114, 1954.

[27] C. J. G. Plummer, L. Garamszegi, Y. Leterrier, M. Rodlert, J. A. E. Månson, Chem. Mater. 2002, 14, 486. [28] F. Hussain, M. Hojjati, M. Okamoto, R. E. Gorga, J. Compos. Mater. 2006, 40, 1511.

[29] M. Rodlert, C. J. G. Plummer, L. Garamszegi, Y. Leterrier, H. J. M. Grunbauer, J. A. E. Månson, Polymer 2004, 45, 949.

[30] M. L. Sham, J. K. Kim, Compos .Part A-Appl. Sci. 2004, 35, 537.

[31] K. Cottier, M. Wiki, G. Voirin, H. Gao, R. E. Kunz, Sensor. Actuat. A-Chem. 2003, 91, 241.

[32] L. E. Schmidt, Y. Leterrier, J. M. Vesin, M. Wilhelm, J. A. E. Månson, Macromol. Mater. Eng. 2005, 290, 1115. [33] Y. Inoue, Y. Kobatake, Appl. Sci. Res. 1958, A7, 314. [34] M. Dewaele, D. Truffier-Boutry, J. Devaux, G. Leloup, Dent. Mater. 2006, 22, 359.

[35] J. Jakubiak, L. A. Linden, Polimery 2001, 46, 522. [36] M. P. Patel, M. Braden, K. W. M. Davy, Biomaterials 1987, 8, 53.

[37] M. F. Witzel, R. Y. Ballester, J. B. C. Meira, R. G. Lima, R. R. Braga, Dent. Mater. 2007, 23, 204.

[38] G. A. Laughlin, J. L. Williams, J. D. Eick, J. Biomed. Mater. Res. 2002, 63, 671.

[39] B. A. Davis, P. J. Gramman, "Compression molding", Hanser Verlage, 2003.

[40] J. A. Payne, L. F. Francis, A. V. McCormick, J Appl. Polym. Sci. 1997, 66, 1267.

[41] D. M. Vaessen, F. A. Ngantung, M. L. B. Palacio, L. F. Francis, A. V. McCormick, J Appl. Polym. Sci. 2002, 84, 2784.

[42] R. Feng, R. J. Farris, J. Micromech. Microeng. 2003, 13,80 .

[43] B. Li, M. Liu, O. F. Chen, J. Microlith. Microfab. 2005, 4. 